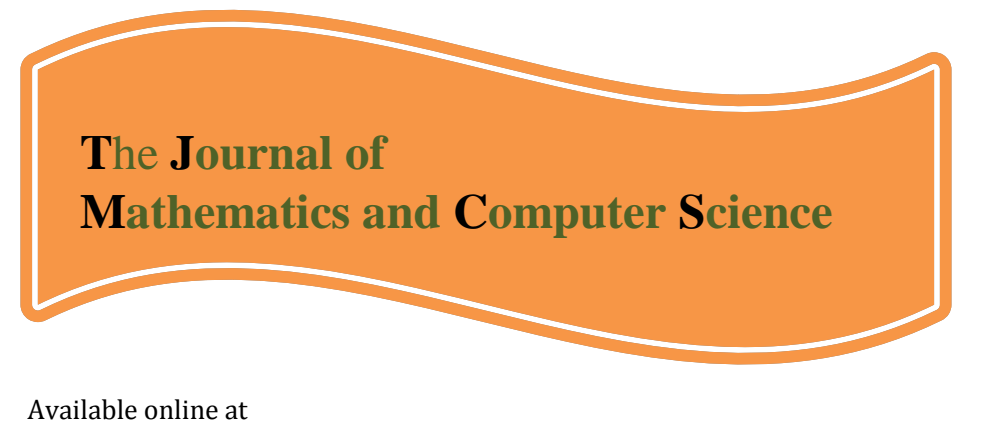

\title{
http://www.TJMCS.com
}

The Journal of Mathematics and Computer Science Vol .4 No.4 (2012) 527-535

\section{The Block AOR Iterative Methods for Solving Fuzzy Linear Systems}

\author{
H. Saberi Najafi \\ Department of Mathematics, Lahijan Branch, Islamic Azad University, Lahijan, Iran \\ e-mail : hnajafi@guilan.ac.ir \\ S.A. Edalatpanah * \\ Department of Mathematics, Lahijan Branch, Islamic Azad University, Lahijan, Iran \\ Young Researchers Club, Lahijan Branch, Islamic Azad University, Lahijan, Iran \\ email: saedalatpanah@gmail.com
}

Received: July 2011, Revised: November 2012

Online Publication: November 2012

* Corresponding author.

\begin{abstract}
In this article the block AOR Iterative methods are used for solving fuzzy linear systems. The convergence of the methods and functional relationship between eigenvalues in block AOR is investigated.
\end{abstract}

Keywords: Fuzzy linear system (FLS); AOR method;Block AOR; Eigenvalue.

\section{Mathematics Subject Classification: $65 F 10$.}

\section{Introduction.}

Unfailing real world problems in economics, finance, mechanics etc. can lead to solving a system of linear equations. There are many methods for solving linear systems, see $[3,11-12,14-15,18]$ and the references therein. Nevertheless, when coefficients of a system are ambiguous and there is some inexplicit information about the exact amount of parameters, how can the linear equation system be solved? To solve this problem, first attempt researchers made was through accidental events and caring to probabilities. However, yet, scientists believe that the only way to improve efficiency is to increase accuracy. Thus, fuzzy logic was proposed by Zadeh in1965 [13,20]. The solutions of fuzzy linear systems (FLS) have been considered by many researchers, for example [1,4-5]. In [4] , Friedman et al. use the embedding method and replaced the fuzzy linear system by a 
$2 \mathrm{n} \times 2 \mathrm{n}$ crisp linear system and studied the uniqueness of the fuzzy solution. This model has been modified later by some other researchers; see [2-3,8-10,16-17] and the references therein. In this article we consider the block AOR iterative methods to solve the fuzzy linear system.

\section{2 .Preliminaries}

In this section we provide some basic notations and definitions of fuzzy number and fuzzy linear system.

\section{Definition 2.1.}

An arbitrary fuzzy number is represented, in parametric form, by an ordered pair of functions $(\underline{u}(r), \bar{u}(r)), 0 \leq r \leq 1$, which satisfy the following requirements(see [1,4-5]):

(i) $\underline{u}(r)$ is a bounded monotonic increasing left continuous function over[0,1];

(ii) $\bar{u}(r)$ is a bounded monotonic decreasing left continuous function over[0,1];

(iii) $\underline{u}(r) \leq \bar{u}(r), 0 \leq r \leq 1$.

A crisp number $\alpha$ can be simply expressed as $\underline{u}(r)=\bar{u}(r)=\alpha, 0 \leq r \leq 1$.

The addition and scalar multiplication of fuzzy numbers previously can be described as follows:

(i) $\mathrm{x}=\mathrm{y}$ if and only if $\underline{x}(r)=\underline{y}(r)$ and $\bar{x}(r)=\bar{y}(r)$,

(ii) $\mathrm{x}+\mathrm{y}=(\underline{x}(r)+\underline{y}(r), \bar{x}(r)+\bar{y}(r))$,

(iii) $K x=\left\{\begin{array}{ll}(K \underline{x}, K \bar{x}), & K \geq 0, \\ (K \bar{x}, K \underline{x}), & K<0,\end{array} \quad K \in R\right.$.

Definition 2.2. Consider the $\mathrm{n} \times \mathrm{n}$ linear system of equations:

$$
\left\{\begin{array}{l}
a_{11} x_{1}+a_{12} x_{2}+\cdots+a_{1 n} x_{n}=b_{1}, \\
a_{21} x_{1}+a_{22} x_{2}+\cdots+a_{2 n} x_{n}=b_{2}, \\
\vdots \\
a_{n 1} x_{1}+a_{n 2} x_{2}+\cdots+a_{n n} x_{n}=b_{n}
\end{array}\right.
$$

Where the coefficient matrix $\mathrm{A}=\left(a_{i j}\right), 1 \leq \mathrm{i}, \mathrm{j} \leq \mathrm{n}$ is a crisp matrix and $b_{i} \in E^{1} ; 1 \leq i \leq n$, is called a fuzzy linear system(FLS).

Definition 2.3. A fuzzy number vector $X=\left(x_{1}, x_{2}, \ldots, x_{n}\right)^{T}$, given by parametric form

$$
X_{i}=\left(\underline{x_{i}}(r), \overline{x_{i}}(r)\right), \quad 1 \leq i \leq n, 0 \leq r \leq 1 \text {, }
$$

is called a solution of the fuzzy linear system (1) if

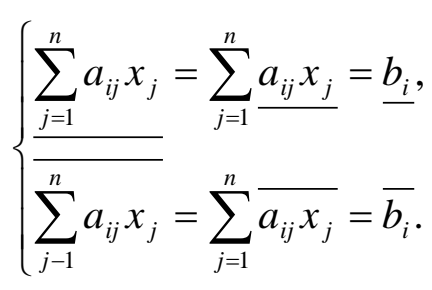


Friedman et al.[4-5], in order to solve the system given by(2) have solved a $2 \mathrm{n} \times 2 \mathrm{n}$ crisp linear system as:

$$
S X=B
$$

where $S=\left(S_{i j}\right)$,are determined as follows;

$$
\left\{\begin{array}{l}
a_{i j} \geq 0 \rightarrow s_{i j}=a_{i j}, \quad s_{i+n, j+n}=a_{i j}, \\
a_{i j}<0 \rightarrow s_{i, j+n}=-a_{i j}, \quad s_{i+n, j}=-a_{i j},
\end{array}\right.
$$

and any $\left(s_{i j}\right)$ which is not determined by $(4)$ is zero.

Then refer to [4] we have ;

$$
\begin{aligned}
& S=\left[\begin{array}{ll}
s_{1} & s_{2} \\
s_{2} & s_{1}
\end{array}\right] \& X=\left[\begin{array}{c}
\underline{x} \\
-\bar{x}
\end{array}\right] \& B=\left[\begin{array}{c}
\underline{b} \\
-\bar{b}
\end{array}\right] \Rightarrow\left\{\begin{array}{l}
s_{1} \underline{x}-s_{2} \bar{x}=\underline{b}, \\
s_{2} \underline{x}-s_{1} \bar{x}=-\bar{b} .
\end{array}\right. \\
& \text { or } \\
& S=\left[\begin{array}{cc}
s_{1} & -s_{2} \\
-s_{2} & s_{1}
\end{array}\right] \& X=\left[\begin{array}{l}
\underline{x} \\
\bar{x}
\end{array}\right] \& B=\left[\begin{array}{l}
\underline{b} \\
\bar{b}
\end{array}\right] \Rightarrow\left\{\begin{array}{l}
s_{1} \underline{x}-s_{2} \bar{x}=\underline{b}, \\
-s_{2} \underline{x}+s_{1} \bar{x}=\bar{b} .
\end{array}\right.
\end{aligned}
$$

Where $s_{1}, s_{2} \geq 0, A=s_{1}-s_{2}$.

Theorem 2.1.[4]. The matrix $S$ is nonsingular if and only if the matrices $A$ and $s_{1}+S_{2}$ are both nonsingular.

Theorem 2.2. [4]. If $S^{-1}$ exists it must have the same structure as $S$, i.e. $S^{-1}=\left[\begin{array}{ll}E & F \\ F & E\end{array}\right]$.

Theorem 2.3.[4]. Let $\mathrm{S}$ be nonsingular. Then the unique solution $X$ is always a fuzzy vector for arbitrary vector $B$, if and only if $S^{-1}$ is nonnegative.

Theorem 2.4. [7]. The inverse of a nonnegative matrix $A$ is nonnegative if and only if $A$ is a generalized permutation matrix.

Definition 2.4[4]. Let denote $X=\left(x_{i}(r), \overline{x_{i}}(r)\right), \quad 1 \leq i \leq n$ the unique solution of $S X=B$. The fuzzy number vector $U=\left(\underline{u_{i}}(r), \overline{u_{i}}(r)\right)$ defined by

$$
\begin{aligned}
& \underline{u_{i}}(r)=\min \left\{\underline{x_{i}}(r), \overline{x_{i}}(r), \underline{x_{i}}(1)\right\}, \\
& \overline{u_{i}}(r)=\max \left\{\underline{x_{i}}(r), \overline{x_{i}}(r), \underline{x_{i}}(1)\right\},
\end{aligned}
$$

is called the fuzzy solution of $S X=B$.

If $X=\left(\underline{x_{i}}(r), \overline{x_{i}}(r)\right)$ are all triangular fuzzy numbers then $\underline{u_{i}}(r)=\underline{x_{i}}(r), \overline{u_{i}}(r)=\overline{x_{i}}(r)$ and $U$ is called a strong fuzzy solution. Otherwise is a weak fuzzy solution .

\section{The block AOR iterative method for fuzzy linear systems}


Let $S$ be nonsingular,$S_{\mathrm{ii}} \neq 0$ and $S=D-L-U$, where;

$$
D=\left[\begin{array}{ll}
D_{1} & 0 \\
0 & D_{1}
\end{array}\right], L=\left[\begin{array}{cc}
L_{1} & 0 \\
S_{2} & L_{1}
\end{array}\right], U=\left[\begin{array}{cc}
U_{1} & S_{2} \\
0 & U_{1}
\end{array}\right]
$$

And,

$$
D_{1}=\operatorname{diag}\left(s_{i, i}\right), i=1,2, \ldots, n \quad, D_{1}-L_{1}-U_{1}=S_{1},
$$

and $L_{1}, U_{1}$ are strictly lower and upper triangular matrices ,respectively.

The iterative method for $S X=B$ is;

$$
X^{(i+1)}=M^{-1} N X^{(i)}+M^{-1} b, \quad i=0,1 \ldots
$$

where $S=M-N$, $\operatorname{det}(\mathrm{M}) \neq 0$ and $X^{(0)}$ is any initial vector. The method is convergent if $\rho\left(M^{-1} N\right)<1$ (we denote the spectral radius of $S$ by $\rho(\mathrm{S})$ ).

In the following, first, we will survey Non-Block AOR method for FLS. This method is defined in[6] and for FLS is used in [2] .

This method is given by the following;

$$
X^{(i+1)}=L_{r, w} X^{(i)}+(D-r L)^{-1} w b, \quad i=0,1, \ldots
$$

and the iterative matrix is;

$$
L_{r, w}=(D-r L)^{-1}[(1-w) D+(w-r) L+w U],
$$

where $(w, r)$ are real parameters with $w \neq 0$.

Then for solving $F L S$, by some algebra and $X^{i}=\left[\begin{array}{c}\underline{x}^{i} \\ \bar{x}^{i}\end{array}\right], k=\left(D_{1}-r L_{1}\right)^{-1}$ we have;

$$
\left\{\begin{aligned}
\underline{x}^{i+1}= & k\left[(1-w) D_{1}+(w-r) L_{1}+w U_{1}\right] \underline{x}^{i}+w k s_{2} \bar{x}^{i}+w k \underline{b} \\
\bar{X}^{i+1} & =w k s_{2} k\left[(1-r) D_{1}+r U_{1}\right] \underline{x}^{i}+k\left[(1-w) D_{1}\right. \\
& \left.+(w-r) L_{1}+r w s_{2} k s_{2}+w U_{1}\right] \bar{x}+r w k s_{2} k \underline{b}+w k \bar{b} .
\end{aligned}\right.
$$

And the iterative matrix for AOR in FLS is;

$$
L_{r, w}=M^{-1} N=\left(\begin{array}{cc}
k & 0 \\
r k s_{2} k & k
\end{array}\right)\left\{\left(\begin{array}{cc}
(1-w) D_{1} & 0 \\
0 & (1-w) D_{1}
\end{array}\right)+\left(\begin{array}{cc}
(w-r) L_{1} & 0 \\
(w-r) s_{2} & (w-r) L_{1}
\end{array}\right)+\left(\begin{array}{ll}
w U_{1} & w s_{2} \\
0 & w U_{1}
\end{array}\right)\right\}
$$

Dehghan and Hashemi in[2] proposed sufficient condition for the convergence of the AOR method when $S$ is a strictly diagonally dominant.(see[2,Theorem4.8]).The following theorem indicates a necessary condition for the convergence of this method, in generally .

This theorem has been proved in [19] for a crisp linear system. Here we prove this theorem for FLS and constructed matrix $S$.

\section{Theorem3.1}

If the AOR method for solving FLS, converges for some $w, r \neq 0$. Then we have only one of the following expressions:

(i) $r \in(0,2) \& w \in(-\infty, 0) \cup(0,+\infty)$, 
(ii) $r \in(-\infty, 0) \cup[2,+\infty) \& w \in(2 r /(2-r), 0) \cup(0,2)$.

And when $r=0$, for convergence we must have $0<\mathrm{w}<2$.

Proof. If in (11), $r=w$ then the iterative matrix of AOR is SOR, i.e. $T_{S O R}$, then for convergence we have:

$$
L_{r, r}=T_{S O R}=\left[\begin{array}{lc}
k\left[(1-r) D_{1}+r U_{1}\right] & r k s_{2} \\
r k s_{2} k\left[(1-r) D_{1}+r U_{1}\right] & k\left[(1-r) D_{1}+r^{2} s_{2} k s_{2}+r U_{1}\right]
\end{array}\right]
$$

Where,

$$
k=\left(D_{1}-r L_{1}\right)^{-1} \text {. }
$$

And with elementary row operations determinant of $L_{r r}$, we have;

$$
\begin{aligned}
& \operatorname{det}\left(L_{r, r}\right)=\operatorname{det}\left(\left[\begin{array}{cc}
k\left[(1-r) D_{1}+r U_{1}\right] & k s_{2} \\
0 & k\left[(1-r) D_{1}+w U_{1}\right]
\end{array}\right]\right) \\
& =\operatorname{det}\left[(1-r) D_{1}+r U_{1}\right] \operatorname{det}\left[(1-r) D_{1}+r U_{1}\right]=(1-r)^{2 n}=\prod_{i=1}^{2 n} \xi_{i} .
\end{aligned}
$$

Where $\xi_{i}$ are eigenvalues of iterative matrix SOR. As we know the AOR method for $r \neq 0$ is an extrapolation of SOR method with $\mathrm{t}=\mathrm{w} / \mathrm{r}$. So we have;

$$
\begin{aligned}
& \lambda_{i}=\frac{w}{r} \xi_{i}+(1-w / r), \quad i=1: 2 n \\
& \Rightarrow(1-r)^{2 n}=\prod_{i=1}^{2 n}\left(1-\frac{r}{w}+\frac{r \lambda_{i}}{w}\right),
\end{aligned}
$$

where $\lambda_{i}$ are eigenvalues of iterative matrix AOR. Since $\lambda_{i}<1,(i=1, \ldots, 2 n)$, we have,

$$
\left|(1-r)^{2 n}\right|=\prod_{i=1}^{2 n}\left|\left(1-\frac{r}{w}+\frac{r \lambda_{i}}{w}\right)\right|<\left(1-\frac{r}{w}|+| \frac{r}{w}\right)^{2 n} .
$$

Therefore,

$$
|1-r|<\left(\left|1-\frac{r}{w}\right|+\left|\frac{r}{w}\right|\right)
$$

Now use Theorem2.1 of [19].

Recently, in [8] the block SOR iterative methods are applied for solving the FLS .

Here, we establish the block AOR iterative methods for solving $\mathrm{n} \times \mathrm{n}$ fuzzy linear system.

The Block form of AOR can be written in different forms. Some of them are as follows;

$$
S=D-L-U,
$$

where,

$$
\begin{aligned}
& D=\left[\begin{array}{lr}
s_{1}-s_{2} & 0 \\
0 & s_{1}-s_{2}
\end{array}\right], L=\left[\begin{array}{ll}
0 & 0 \\
s_{2} & 0
\end{array}\right], U=\left[\begin{array}{cc}
-s_{2} & s_{2} \\
0 & -s_{2}
\end{array}\right], \\
& D=\left[\begin{array}{ll}
s_{1}-s_{2} & 0 \\
0 & s_{1}+s_{2}
\end{array}\right], L=\left[\begin{array}{cc}
-s_{2} & 0 \\
s_{2} & s_{2}
\end{array}\right], U=\left[\begin{array}{cc}
0 & s_{2} \\
0 & 0
\end{array}\right],
\end{aligned}
$$




$$
D=\left[\begin{array}{lr}
s_{1}+s_{2} & 0 \\
0 & s_{1}+s_{2}
\end{array}\right], L=\left[\begin{array}{ll}
0 & 0 \\
s_{2} & 0
\end{array}\right], U=\left[\begin{array}{cc}
s_{2} & s_{2} \\
0 & s_{2}
\end{array}\right] .
$$

For splitting form of (12) we have,

$$
X^{(i+1)}=T_{r, w} X^{(i)}+(D-r L)^{-1} w b, \quad i=0,1, \cdots
$$

where,

$$
\begin{aligned}
& X^{(i)}=\left(\underline{x}^{(i)}(r), \bar{x}^{(i)}(r)\right), \\
T_{r, w}=(D-r L)^{-1}[(1-w) D+(w-r) L+w U], & \\
= & {\left[\begin{array}{ll}
s_{1}-s_{2} & 0 \\
-r s_{2} & s_{1}-s_{2}
\end{array}\right]^{-1} \times\left[\begin{array}{cc}
(1-w)\left(s_{1}-s_{2}\right)-w s_{2} & w s_{2} \\
-(w-r) s_{2} & (1-w)\left(s_{1}-s_{2}\right)-w s_{2}
\end{array}\right] . }
\end{aligned}
$$

Furthermore, the stopping criterion with tolerance $\varepsilon \succ 0$ is;

$$
\frac{\left\|x^{i+1}-\bar{x}\right\|}{\left\|x^{i+1}\right\|}<\varepsilon \& \frac{\left\|\underline{x}^{i+1}-\underline{x}^{i}\right\|}{\left\|\underline{x}^{i+1}\right\|}<\varepsilon . \quad i=0,1, \ldots
$$

And for any triangular fuzzy number $x=(a+b r, c+d r), 0 \leq r \leq 1$, its norm is defined as;

$$
\|x\|=\max \{|a|,|b|,|c|,|d|\} .
$$

Where this is equivalent to Hausdorff distance of fuzzy numbers.

Some functional relations between the eigenvalues of the iterative matrices for the block SOR iterative methods are proposed in [8] . In the following theorem we present the functional relation between the eigenvalues $\lambda\left(T_{r, w}\right)$ of the iterative matrix(16) for the block AOR iterative method. Similar functional relations for different Block forms of AOR can be obtained in the same manner. Theorem 3.2. For Block AOR form of (12) , we have the following functional relationship

$$
(1-w-\lambda)^{2}=2 w(1-w-\lambda) \mu+r w(\lambda-1) \mu^{2}
$$

In which $\lambda$ is any eigenvalue of iterative matrix $T_{r, w}$ except $\lambda=1-w$,and $\mu$ is any eigenvalue of $\left(s_{1}-s_{2}\right)^{-1} s_{2}$.

Proof. By refer to the proof of theorem 3.1,if in iterative matrix AOR of (11), r=w then we have the iterative matrix SOR. Now let $\xi$ be the nonzero eigenvalues of $T_{r, r}$ and $\left(x^{t}, y^{t}\right)^{t}$ be the corresponding eigenvector, then we have ;

$$
\begin{aligned}
& T_{r, r}\left(\begin{array}{l}
x \\
y
\end{array}\right)=\xi\left(\begin{array}{l}
x \\
y
\end{array}\right), \\
& \Rightarrow\left[\begin{array}{cc}
(1-r)\left(s_{1}-s_{2}\right)-r s_{2} & r s_{2} \\
0 & (1-r)\left(s_{1}-s_{2}\right)-r s_{2}
\end{array}\right]\left(\begin{array}{l}
x \\
y
\end{array}\right)=\xi\left[\begin{array}{ll}
s_{1}-s_{2} & 0 \\
-r s_{2} & s_{1}-s_{2}
\end{array}\right]\left(\begin{array}{l}
x \\
y
\end{array}\right) .
\end{aligned}
$$

It is easy to see that,

$$
\left\{\begin{array}{l}
(1-r-\xi)\left(s_{1}-s_{2}\right) x=r s_{2} x-r s_{2} y \\
(1-r-\xi)\left(s_{1}-s_{2}\right) y=r s_{2} y-r \xi s_{2} x
\end{array}\right.
$$

Since $A$ is nonsingular then $\left(s_{1}-s_{2}\right)$ is nonsingular and also $\xi \neq 1-r$ therefore from (18) we have, 


$$
(1-r-\xi)^{2} x=2 r(1-r-\xi)\left(s_{1}-s_{2}\right)^{-1} s_{2} x+r^{2}(\xi-1)\left(\left(s_{1}-s_{2}\right)^{-1} s_{2}\right)^{2} x
$$

Thus,

$$
(1-r-\xi)^{2}=2 r(1-r-\xi) \mu+r^{2}(\xi-1) \mu^{2}
$$

From (20) one can see that if $\mu$ be the eigenvalue of $\left(s_{1}-s_{2}\right)^{-1} s_{2}$ then $\xi$ is an eigenvalue of $T_{r, r}$ and vice versa. Now since $t=w / r$ and $L_{r, w}=t L_{r, r}+(1-t) I$ if $\lambda$ is an eigenvalue of iterative matrix of AOR then the proof is completed.

We also could have similar theorems for (13),(14).

Finally, in order to show that the block AOR iterative methods are efficient for the fuzzy linear systems, consider the following $3 \times 3$ fuzzy system

$$
\left\{\begin{array}{l}
x_{1}+2 x_{2}-x_{3}=(-1+2 r, 11-10 r), \\
2 x_{1}-x_{2}+x_{3}=(10+4 r, 16-2 r), \\
x_{1}+2 x_{2}+3 x_{3}=(14+6 r, 32-12 r) .
\end{array}\right.
$$

The extended $6 \times 6$ matrix is;

$$
S=\left[\begin{array}{cc}
s_{1} & -s_{2} \\
-s_{2} & s_{1}
\end{array}\right]=\left[\begin{array}{cccccc}
1 & 2 & 0 & 0 & 0 & -1 \\
2 & 0 & 1 & 0 & -1 & 0 \\
1 & 2 & 3 & 0 & 0 & 0 \\
0 & 0 & -1 & 1 & 2 & 3 \\
0 & -1 & 0 & 2 & 0 & 1 \\
0 & 0 & 0 & 1 & 2 & 3
\end{array}\right]
$$

Which $S, s_{1}$ are invertible. The exact solution is;

$$
\left\{\begin{array}{l}
x_{1}=(1+3.25 r, 4+0.25 r) \\
x_{2}=(2-1.25 r, 5-4.25 r) \\
x_{3}=(3+1.75 r, 6-1.25 r)
\end{array}\right.
$$

This is a strong fuzzy solution. In this FLS, since iteration matrix in Non-Block AOR is singular, these point methods cannot be used in the above FLS. But our methods work well. The following table shows the numerical results of above example with the tolerance ( $\varepsilon=0.00001$ ) and $X_{0}=\left[\begin{array}{llllll}0 & 0 & 0 & 0 & 0 & 0\end{array}\right]^{T}$. In the Table 1, we reported the number of iterations (Iter) and the spectral radius $(\rho)$ of the corresponding iterative matrix with different parameters $\mathrm{w}, \mathrm{r}$. Furthermore, (\#) indicates the method is not convergence.

Table1 Shows the results of example for Block AOR

\begin{tabular}{cc|cc|cc|cc}
\hline \multicolumn{2}{c|}{ Method } & \multicolumn{2}{c|}{ lock form of(12) } & \multicolumn{2}{c|}{ Block form of(13) } & \multicolumn{2}{c}{ Block form of(14) } \\
\hline $\mathrm{w}$ & $\mathrm{r}$ & $\boldsymbol{\rho}$ & Iter & $\boldsymbol{\rho}$ & Iter & $\boldsymbol{\rho}$ & Iter \\
\hline 0.1 & 0.3 & 0.9480 & 151 & 0.9336 & 128 & 0.9380 & 151 \\
1.0 & 0.0 & 0.6667 & 31 & 0.6404 & 29 & 1.0000 & $\#$ \\
0.8 & 0.5 & 0.5711 & 14 & 0.4342 & 11 & 0.4922 & 14 \\
1.1 & 1.0 & 0.4534 & 18 & 0.1000 & $\mathbf{7}$ & 0.6285 & 29 \\
1.5 & 1.5 & 1.0000 & $\#$ & 0.5000 & 21 & 1.2500 & $\#$
\end{tabular}


In the following we can to see that the approximate solution above example for $(w=0.8, r=0.5)$.

Block form of(12); $\left\{\begin{array}{l}x_{1}=(0.9995+3.2496 r, 4.0004+0.2496 r), \\ x_{2}=(1.9998-1.2500 r, 5.0002-4.2500 r), \\ x_{3}=(3.0003+1.7500 r, 5.9997-1.2500 r) .\end{array}\right.$

Block form of(13); $\left\{\begin{array}{l}x_{1}=(1.0002+3.2501 r, 4.0004+0.2497 r), \\ x_{2}=(1.9998-1.2498 r, 4.9999-4.2500 r), \\ x_{3}=(3.0001+1.7498 r, 6.0000-1.2499 r) .\end{array}\right.$

Block form of $(14) ;\left\{\begin{array}{l}x_{1}=(1.0004+3.2501 r, 4.0003+0.2501 r), \\ x_{2}=(1.9998-1.2500 r, 4.9998-4.2499 r), \\ x_{3}=(3.0000+1.7499 r, 6.0000-1.2500 r) .\end{array}\right.$

\section{Acknowledgment.}

The authors would like to thank professor F.G. Lupianes, the editor of the JMCS journal for the valuable help and cooperation and also to thank the referees of this article for their great suggestions which lead to the publishing of our article.

\section{References .}

[1] J .J. Buckley and Y.Qu, Solving fuzzy equations: a new solution concept, Fuzzy Sets and Systems 39 (1991) 291-301.

[2] M. Dehghan, B. Hashemi, Iterative solution of fuzzy linear systems, Appl. Math. Comput. 175 (2006),645-674.

[3] S. A. Edalatpanah, The preconditioning AOR method for solving linear equation systems with iterative methods, Dissertation, Lahijan Branch, Islamic Azad University, 2008.

[4] M . Friedman, Ma. Ming and A . Kandel, Fuzzy linear systems, Fuzzy Sets and Systems 96 (1998), 201-209.

[5] M . Friedman, Ma. Ming and A . Kandel, Duality infuzzy linear systems, Fuzzy Sets and Systems 109 (2000), 55-58.

[6] A. Hadjidimos, Accelerated overrelaxation method, Math. Comput. 32 (1978), 149-157.

[7] H. Minc, Nonnegative Matrices ,Wily, New York, 1998.

[8] S. Miao, B. Zheng ,K.Wang, Block SOR methods for fuzzy linear systems, J Appl Math Comput 26(2008),201-218.

[9] S.H. Nasseri, F. Zahmatkesh, Huang method for solving fully fuzzy linear system , The Journal of Mathematics and Computer Science,1(2010),1-5.

[10] S.H. Nasseri, M. Gholami, Linear System of Equations with Trapezoidal Fuzzy Numbers, The Journal of Mathematics and Computer Science ,3(2011),71-79.

[11] H. Saberi Najafi, S. Kordrostami and S.A .Edalatpanah, The Convergence Analysis Of Preconditioned AOR Method For M-, H-Matrices, Journal of Applied Mathematics IAUL , 
$5(2008), 29-38$.

[12] H.Saberi Najafi and S.A. Edalatpanah, Fast Iterative Method-FIM.Application to the ConvectionDiffusion Equation, Journal of Information and Computing Science , 6 (2011), 303-313.

[13] H. Saberi Najafi ,F. Ramezani Sasemasi, S. Sabouri Roudkoli, S. Fazeli Nodehi, Comparison of two methods for solving fuzzy differential equations based on Euler method and Zadeh's extension, The Journal of Mathematics and Computer Science,2(2011),295-306.

[14] H. Saberi Najafi and S.A. Edalatpanah, Some Improvements In Preconditioned Modified Accelerated Overrelaxation (PMAOR) Method For Solving Linear Systems, Journal of Information and Computing Science , 6(2011),15-22.

[15] H. Saberi Najafi and S.A. Edalatpanah , On the Convergence Regions of Generalized AOR Methods for Linear Complementarity Problems, Journal of Optimization Theory and Applications,(2012), DOI 10.1007/s10957-012-0135-1.

[16] H.Saberi Najafi,S.A.Edalatpanah, An Improved Model for Iterative Algorithms in Fuzzy Linear Systems , Computational Mathematics and Modeling ,accepted.

[17] H.Saberi Najafi, S.A.Edalatpanah, Preconditioning Strategy to Solve Fuzzy Linear Systems (FLS), International Review of Fuzzy Mathematics, accepted.

[18] R.S. Varga ,Matrix Iterative Analysis. second ed. Springer,Berlin,2000.

[19] A.k. Yeyios, A necessary condition for the convergence of the accelerated overrelaxation (AOR) method, J. Comput. Appl. Math. 26 (1989), 371-373 .

[20] L.A. Zadeh, Fuzzy sets, Information and Control 8 (1965), 338-353. 